\title{
Annotating activation/inhibition relationships to protein-protein interactions using gene ontology relations
}

\author{
Soorin Yim ${ }^{1,2}$, Hasun Yu², Dongjin Jang ${ }^{1}$ and Doheon Lee ${ }^{1,2^{*}}$ \\ From The Sixteenth Asia Pacific Bioinformatics Conference \\ Yokohama, Japan. 15-17 January 2018
}

\begin{abstract}
Background: Signaling pathways can be reconstructed by identifying 'effect types' (i.e. activation/inhibition) of protein-protein interactions (PPIs). Effect types are composed of 'directions' (i.e. upstream/downstream) and 'signs' (i.e. positive/negative), thereby requiring directions as well as signs of PPIs to predict signaling events from PPI networks. Here, we propose a computational method for systemically annotating effect types to PPIs using relations between functional information of proteins.

Results: We used regulates, positively regulates, and negatively regulates relations in Gene Ontology (GO) to predict directions and signs of PPIs. These relations indicate both directions and signs between GO terms so that we can project directions and signs between relevant GO terms to PPIs. Independent test results showed that our method is effective for predicting both directions and signs of PPIs. Moreover, our method outperformed a previous GO-based method that did not consider the relations between GO terms. We annotated effect types to human PPIs and validated several highly confident effect types against literature. The annotated human PPIs are available in Additional file 2 to aid signaling pathway reconstruction and network biology research.

Conclusions: We annotated effect types to PPIs by using regulates, positively regulates, and negatively regulates relations in GO. We demonstrated that those relations are effective for predicting not only signs, but also directions of PPIs. The usefulness of those relations suggests their potential applications to other types of interactions such as protein-DNA interactions.
\end{abstract}

Keywords: Protein-protein interaction, Activation, Inhibition, Gene ontology

\section{Background}

A cell reacts to stimuli through signaling pathways, in which proteins physically interact with each other to transmit signals. Those signals propagate inside a cell, causing various responses such as cell proliferation and differentiation [1-4]. Abnormal signal transduction triggers aberrant biological processes that might result in diseases such as cancer [2-5]. To understand how such signals flow, various high-throughput experiments have been developed

\footnotetext{
* Correspondence: dhlee@biosoft.kaist.ac.kr

'Department of Bio and Brain Engineering, KAIST, 291 Daehak-ro,

Yuseong-gu, Daejeon 34141, Republic of Korea

²Bio-Synergy Research Center, 291 Daehak-ro, Yuseong-gu, Daejeon 34141, Republic of Korea
}

to detect protein-protein interactions (PPIs) such as yeast two hybrid and affinity purification-mass spectroscopy [6]. Even though such high-throughput experiments can determine whether two proteins bind to each other or not, they are not sufficient for reconstructing signaling pathways.

To reconstruct signaling pathways from PPI networks, we need to know two aspects of PPIs: 'directions', and 'signs'. Directions of PPIs represent upstream/downstream relationships, indicating the direction of signal flow. Signs of PPIs represent whether the interactions have positive effects or negative effects. By combining directions with signs, we can define activation/inhibition relationships of PPIs, which we call 'effect types'. 
Effect types are indispensable for not only reconstructing signaling pathways, but also other research areas such as network pharmacology [7, 8]. Without directions, we cannot know causality. This leads to many false positive results that arise from mistaking an effect as a cause [8]. Without signs, we cannot distinguish whether a result is desirable or harmful. For example, when signs are unavailable for drug-disease associations, we cannot differentiate whether a drug cures a disease, or causes a disease as a side effect [9].

Despite effect types are important, no experimental method is available that determines effect types of PPIs in a high-throughput way. Satisfying this need, several computational methods have been proposed to predict signs of PPIs systemically [10-12]. Based on data they used, previous works can be categorized into phenotype-based methods $[10,11]$ and a Gene Ontology (GO) [13]-based method [12]. Phenotype-based methods used RNA interference (RNAi) screening to identify phenotypes that were affected by a gene knockdown. Then, they predicted signs of PPIs based on the hypothesis that proteins resulting in similar phenotypes would interact positively [10, 11]. Even though they were effective, they have two limitations. Firstly, they ignored directions even though their aim was predicting effect types. Secondly, predicted signs cannot be generically applied to human PPIs. Because conducting RNAi screening for all proteins is experimentally expensive, they applied their method to smaller Drosophila melanogaster [10], or HeLa cells [11].

To overcome these limitations, a recent method utilized GO, which is more directly related to proteins [12]. Their hypothesis was that proteins with similar GO annotations would interact positively. They used GO terms as features for representing PPIs, and trained L2-regularized logistic regression model. Even though they improved the performance by using more direct data, they have mainly three limitations. Firstly, still they did not consider direction, leaving the causality between two proteins unknown. Secondly, similar GO annotations not necessarily means two proteins interact positively. In either positive PPIs or negative PPIs, two proteins interact with each other in any case. Therefore, negatively interacting proteins might also participate in the same biological process or have similar molecular function. In fact, the exactly same feature encoding was used for predicting whether two proteins interact or not, treating positive PPIs and negative PPIs equally [13]. Thirdly, they did not consider GO relations. However, GO has positively regulates and negatively regulates relations, which indicate signs between GO terms. These relations might help to differentiate negative PPIs in which one protein negatively regulates a biological process in which the other protein participates. Moreover, those relations indicate directions between GO terms, suggesting their potential use in predicting effect types of molecular interactions.

Here, we propose a method for annotating directions as well as signs to PPIs. We hypothesized that directions and signs between $\mathrm{GO}$ terms, represented by regulates, positively regulates, and negatively regulates relations, can be used for predicting directions and signs of PPIs. The rationale behind this hypothesis is as follows. Let us assume that protein $\mathrm{p} 1$ and $\mathrm{p} 2$ interact with each other, and there is a significant tendency in which GO terms involving $\mathrm{p} 1$ positively regulates $\mathrm{GO}$ terms involving $\mathrm{p} 2$. Since protein $\mathrm{p} 1$ and $\mathrm{p} 2$ interact with each other, the tendency of positive regulation might result from activation of $\mathrm{p} 2$ by $\mathrm{p} 1$. Based on this hypothesis, we predicted directions of undirected, unsigned PPIs first. Then, we predicted sign for each directed, unsigned PPI. PPIs were represented by features that were generated from regulates, positively regulates, and negatively regulates relations. Then, we trained logistic regression models for predicting directions and signs. Independent test results demonstrated that our method outperforms previous GO-based method, especially for negative PPIs. In addition, we annotated effect types to human PPIs and validated highly confident predictions against literature.

\section{Methods \\ Method overview}

The overall method for annotating effect types to PPIs is illustrated in Fig. 1. The input was an undirected, unsigned PPI network. For each undirected, unsigned PPI, we predicted its direction first. We trained two logistic regression models that predicted whether a signal can flow in left-to-right direction and right-to-left direction, respectively. The two models shared the same feature vectors, which were composed of pairs of GO terms between which regulates, positively regulates, or negatively regulates relation hold. By combining outputs of these two models, we decided final direction of a PPI as one of 'left-to-right', 'right-to-left', and 'bi-directional'. Then, we predicted sign for each directed, unsigned PPI. If the PPI is bi-directional, we predicted each sign for both directions. For predicting signs, we trained two logistic regression models that predicted whether a directed PPI can act as activation and inhibition, respectively. The two models shared an identical feature vector, which was composed of pairs of GO terms between which positively regulates or negatively regulates relation hold. By combining outputs of these two models, we decided final effect type as one of the followings: 'activation', 'inhibition', 'activation\&inhibition', and 'affect'. As a result, we obtained PPIs with effect type. 


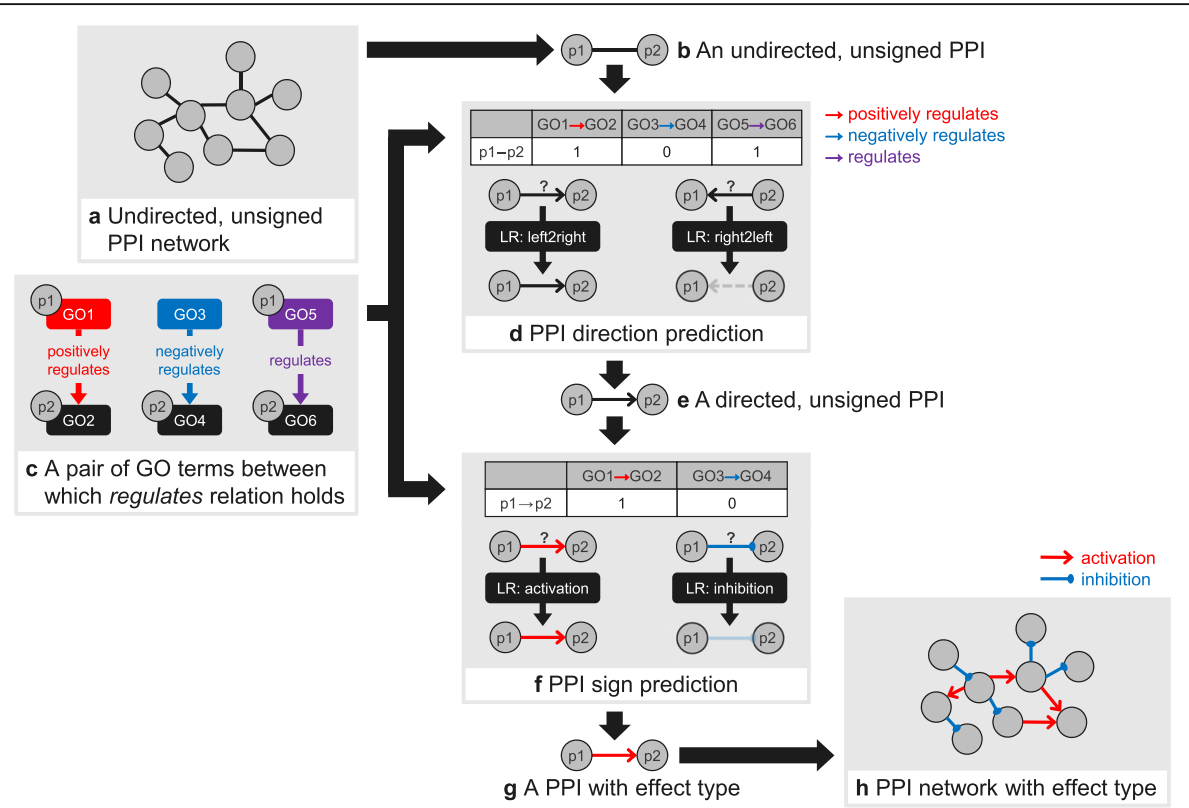

Fig. 1 Method overview. a Input was an undirected, unsigned PPI network. b, c, d For each undirected, unsigned PPI, direction was predicted. We trained two logistic regression models that predicted whether the signal can flow in left-to-right direction and right-to-left direction, respectively. Feature vector was composed of pairs of GO terms between which regulates, positively regulates, or negatively regulates relation holds. e A directed, unsigned PPI was obtained as a result of direction prediction. $\mathbf{f}$ A sign of PPI was predicted for each directed PPI. We trained two logistic regression models that predicted whether a directed PPI can act as activation or inhibition, respectively. Feature vector was composed of a pair of GO terms between which positively regulates or negatively regulates relation holds. $\mathbf{g}, \mathbf{h}$ As a result of direction prediction and sign prediction, we annotated effect types to PPI network. Abbreviations: GO, gene ontology; PPI, protein-protein interaction; LR, logistic regression

\section{Dataset}

\section{PPI dataset}

We collected three PPI datasets: a training set, an independent test set, and a prediction set. To gather reliable datasets, we applied following policies to all datasets: (1) We collected human PPIs only. (2) We removed functional associations and self-interactions. (3) We collected PPIs only when at least one regulates relation holds between GO terms to which constituent proteins are annotated. (4) We mapped protein families to their members. (5) We integrated multiple instances of the same PPI to remove redundancy.

For the training set and the independent test set, we determined directions for each protein pair, and then determined sign for each directed PPI. For proteins protein 1 (p1) and protein 2 (p2), if a signal can flow in only one direction, for example from $\mathrm{p} 1$ to $\mathrm{p} 2$, the direction is 'uni-directional'. On the other hand, if the signal can flow in both directions depending on a context, the direction is 'bi-directional'. Then, we determined sign for each directed PPI. If a PPI is bi-directional, we determined sign for each direction independently. The same directed PPI can act as both activation and inhibition, depending on a context. For example, naked cuticle (NKD) binds to dishevelled segment polarity protein (DVL). This PPI acts as a switch from canonical Wnt signaling pathway to planar cell polarity (PCP) Wnt signaling pathway [14].
This means that NKD inhibits DVL in the aspect of canonical Wnt signaling pathway, whereas NKD activates DVL in the aspect of PCP Wnt signaling pathway. To deal with such context dependency, we categorized effect types of PPIs into four classes: 'activation', 'inhibition', both activation and inhibition are possible depending on a context ('activation\&inhibition'), and neither activation nor inhibition ('affect').

We collected PPIs with known effect types as a training set from Kyoto Encyclopedia of Genes and Genomes (KEGG) pathway [15]. KEGG is a manually curated database for pathways, and contains the largest number of PPIs whose effect types are known. Following the policies, we collected 'PPrels' whose subtypes were one of the followings: activation, inhibition, phosphorylation, dephosphorylation, glycosylation, and methylation.

We gathered another set of PPIs with known effect types as an independent test set from Search Tool for the Retrieval of Interacting Genes/Proteins (STRING) [16]. STRING is an integrated database for proteinprotein associations, including functional and inferred associations. To secure reliable PPIs, we collected PPIs that were experimentally validated. Moreover, we used PPIs whose scores were higher than 800 out of 1000 , which resulted in about $1.28 \%$ of the PPIs available in STRING. In addition, we removed PPIs that were in the training set. 
We collected prediction set from Biological General Repository for Interaction Datasets (BioGRID), whose effect types were previously unknown and predicted by our method [17]. We collected multi-validated PPIs that were validated in at least two experimental systems or two publications. We removed PPIs that were in the training set or the independent test set. As a result, we obtained 20,192 PPIs as a training set, 3420 PPIs as an independent test set, and 28,742 PPIs as a prediction set as shown in Table 1.

\section{GO dataset}

We collected ontologies and GO annotations from GO [18]. We defined a concept of 'regulators' and 'regulatees' for GO terms. A regulator is a GO term that regulates another GO term. If it positively regulates another GO term, then it is a positive regulator, whereas it is a negative regulator if it negatively regulates one. Hereinafter we collectively refer to regulates, positively regulates, and negatively regulates as (positively/negatively) regulates when any of them are applicable. A regulatee is a GO term that is (positively/negatively) regulated by another GO term. For example, 'chromatin silencing' negatively regulates 'transcription, DNA-templated'. Therefore, 'chromatin silencing' is a negative regulator whereas 'transcription, DNA-templated' is a regulatee.

To find all (positive/negative) regulators, we composed GO relations to form a composite relation, such that

relation 1 -relation $2 \rightarrow$ composite relation.

For instance, composing is a with positively regulates becomes positively regulates. Since 'actin nucleation' is a 'positive regulation of actin filament polymerization', which positively regulates 'actin filament polymerization', 'actin nucleation' becomes a positive regulator. This is called 'relation reasoning', and all possible composite relations are listed in Additional file 1: Table S1. We

Table 1 PPI dataset statistics

\begin{tabular}{clllll}
\hline Direction & & & & \\
& Uni-directional & Bi-directional & Total & \\
KEGG & 20,078 & 114 & & 20,192 & \\
STRING & 3110 & 310 & & 3420 & \\
BioGRID & - & - & & 28,742 & \\
Effect type & & & & & \\
& Activation & Inhibition & Activation\& & Affect & Total \\
& & & inhibition & & \\
KEGG & 10,603 & 4548 & 207 & 447 & 15,805 \\
STRING & 2732 & 466 & 133 & 13 & 3344 \\
BioGRID & - & - & - & - & - \\
\hline
\end{tabular}

iteratively applied relation reasoning to find all (positive/ negative) regulators, thereby increase coverage of our method. Hereinafter, we do not differentiate whether a regulator directly regulates regulatee, or indirectly regulates by a composite relation. The statistics of GO terms are shown in Fig. 2. Two hundred fifty six molecular function terms were regulators among 10,940 molecular function terms. For biological process terms, 11,820 terms were regulators out of 29,584 biological process terms.

\section{Feature generation for representing PPIs}

To encode the directions and signs between GO terms, we defined the concept of a $\mathrm{p} 1 \rightarrow \mathrm{p} 2$ (positive/negative) regulation pair. A p1 $\rightarrow \mathrm{p} 2$ (positive/negative) regulation pair is a pair of a (positive/negative) regulator and a corresponding regulatee, in which protein $\mathrm{p} 1$ is annotated to the (positive/negative) regulator and protein $\mathrm{p} 2$ is annotated to the regulatee. For example, if protein p1 is annotated to 'chromatin silencing' and protein p2 is annotated to 'transcription, DNA-templated', then 'chromatin silencing' and 'transcription, DNA-templated' constitute a p1 $\rightarrow$ p2 negative regulation pair as depicted in Fig. 3a.

A p1 $\rightarrow$ p2 (positive/negative) regulation pair indicates direction and sign between GO terms. We projected such directions and signs between GO terms to PPIs, by using each $\mathrm{p} 1 \rightarrow \mathrm{p} 2$ (positive/negative) regulation pair as a feature for representing PPIs. We describe feature generation procedure with a toy example illustrated in Fig. 3b, which is a subset of GO terms related to Wnt signaling pathway.

Features were generated by following procedures. Firstly, we collected all GO terms to which proteins were annotated. In our toy example, protein $\mathrm{p} 1$ is annotated to four GO terms: 'Wnt signaling pathway', 'positive regulation of Wnt signaling pathway', 'regulation of Wnt signaling pathway', and 'positive regulation of canonical Wnt signaling pathway'. On the other hand, protein $\mathrm{p} 2$ is annotated to three GO terms: 'regulation of Wnt signaling pathway,' 'canonical Wnt signaling pathway', and 'negative regulation of canonical Wnt signaling pathway'.

Secondly, for all possible pairs of GO1 and GO2 in which $\mathrm{p} 1$ is annotated to $\mathrm{GO} 1$ and $\mathrm{p} 2$ is annotated to $\mathrm{GO} 2$, we determined whether GO1 (positively/negatively) regulates $\mathrm{GO} 2$. If it did, then we regarded $\mathrm{GO} 1$ and $\mathrm{GO} 2$ as a $\mathrm{p} 1 \rightarrow \mathrm{p} 2$ (positive/negative) regulation pair. However, in many cases, GO1 did not (positively/negatively) regulate GO2 itself. In such cases, we regarded that if $\mathrm{p} 2$ is annotated to GO2, then $\mathrm{p} 2$ is also annotated to ancestors of GO2 that have is a or part of relation with GO2. For example, despite p2 is not directly annotated to 'Wnt signaling pathway', we can say that $\mathrm{p} 2$ is related to 'Wnt signaling pathway' because p2 is annotated to 


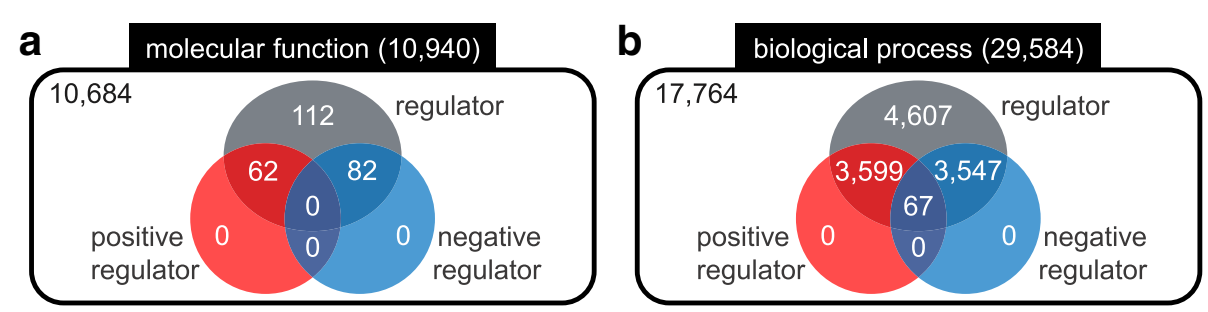

Fig. 2 The statistics of GO terms. a Among 10,940 molecular function terms, 256 terms (2.3\%) were regulators. b There were 11,820 (40.0\%) regulators among 29,584 biological process terms, among which 67 terms were both positive and negative regulator

'canonical Wnt signaling pathway'. This kind of extending GO annotations of a protein by using is $a$ and part of relations in GO is called 'annotation grouping'. To increase our coverage, if GO1 did not (positively/ negatively) regulates $\mathrm{GO} 2$ itself, we applied annotating grouping and determined whether GO1 (positively/ negatively) regulates any ancestors of $\mathrm{GO} 2$. If it did, then we found the most specific ancestor of GO2 with the highest information content that is regulated by GO1 [19]. Then, we regarded GO1 and the most specific ancestor of GO2 that is regulated by GO1 as a $\mathrm{p} 1 \rightarrow \mathrm{p} 2$ (positive/negative) regulation pair. Since excessive annotation grouping might result in too high-dimensional feature vectors in which features are highly correlated, we did not applied annotation grouping when GO1 regulates GO2 itself. For the same reason, we used only the most specific ancestor of $\mathrm{GO} 2$, not all the ancestors.

For example, since $\mathrm{p} 1$ is annotated 'positive regulation of canonical Wnt signaling pathway' and p2 is annotated to 'canonical Wnt signaling pathway', the two GO terms form a $\mathrm{p} 1 \rightarrow \mathrm{p} 2$ positive regulation pair. On the other hand, 'positive regulation of Wnt signaling pathway' does not regulate 'canonical Wnt signaling pathway'. However, p2 is related to 'Wnt signaling pathway' when we apply annotation grouping. Thus, 'positive regulation of Wnt signaling pathway' and 'Wnt signaling pathway' constitute another $\mathrm{p} 1 \rightarrow \mathrm{p} 2$ positive regulation pair.

We repeated the same procedure for determining whether GO2 (positively/negatively) regulates $\mathrm{GO} 1$ or its ancestors. As a result, we found six kinds of regulation pairs: $\mathrm{p} 1 \rightarrow \mathrm{p} 2$ regulation pairs, $\mathrm{p} 1 \rightarrow \mathrm{p} 2$ positive regulation pairs, $\mathrm{p} 1 \rightarrow \mathrm{p} 2$ negative regulation pairs, $\mathrm{p} 2 \rightarrow \mathrm{p} 1$ regulation pairs, $\mathrm{p} 2 \rightarrow \mathrm{p} 1$ positive regulation pairs, and $\mathrm{p} 2 \rightarrow \mathrm{p} 1$ negative regulation pairs. The six kinds of regulation pairs were used as features for predicting directions and signs of PPIs.

\section{Feature generation for predicting directions of PPIs}

For predicting directions of PPIs, we only considered the directions of (positive/negative) regulation pairs; whether it is from $\mathrm{p} 1$ to $\mathrm{p} 2$, or from $\mathrm{p} 2$ to $\mathrm{p} 1$. We did not differentiate regulation pairs, positive regulation pairs, and negative regulation pairs. The value of a (positive/ negative) regulation pair GO1-GO2 is defined as:

$\mathrm{f}_{\text {direction }}[\mathrm{GO} 1-\mathrm{GO} 2]= \begin{cases}1 & \text { if } \mathrm{GO} 1-\mathrm{GO} 2 \text { is a } \mathrm{p} 1 \rightarrow \mathrm{p} 2 \text { (positive/negative) } \\ -1 & \begin{array}{l}\text { regulation pair, exclusively } \\ \text { if } \mathrm{GO} 1-\mathrm{GO} 2 \text { is a } \mathrm{p} 2 \rightarrow \mathrm{p} 1 \text { (positive/negative) }\end{array} \\ 0 & \text { regulation pair, exclusively }\end{cases}$

In our toy example, since 'positive regulation of canonical Wnt signaling pathway' and 'canonical Wnt signaling pathway' constitute a $\mathrm{p} 1 \rightarrow \mathrm{p} 2$ positive regulation pair, but not a $\mathrm{p} 2 \rightarrow \mathrm{p} 1$ positive regulation pair, it has the value of one. On the other hand, 'negative regulation of canonical Wnt signaling pathway' and 'Wnt signaling pathway' form a $\mathrm{p} 2 \rightarrow \mathrm{p} 1$ negative regulation pair, exclusively. Thus, it has the value of -1 . If the direction of a (positive/negative) regulation pair is both from $\mathrm{p} 1$ to $\mathrm{p} 2$ and from $\mathrm{p} 2$ to $\mathrm{p} 1$, then the feature value is zero. In the toy example, since $\mathrm{p} 1 \rightarrow \mathrm{p} 2$ (positive/negative) regulation pairs outnumber $\mathrm{p} 2 \rightarrow \mathrm{p} 1$ (positive/negative) regulation pairs, the direction of PPI is more likely to be from p1 to p2. We removed (positive/negative) regulation pairs that were not used in the training set and the number of features for direction was 37,617.

\section{Feature generation for predicting signs of PPIs}

Feature generation for predicting signs of PPIs are similar to that for directions; We used each regulation pair as a feature. However, there are also some differences: (1) Since we predicted sign for a directed PPI, we used regulation pairs whose directions were consistent with the direction of the PPI. (2) Since simple regulation pairs are uninformative for predicting signs, we used positive regulation pairs and negative regulation pairs only. (3) We removed regulation pairs that is both positive and negative. For example, 'cell cycle switching, mitotic to meiotic cell cycle' positively regulates 'meiotic cell cycle' and negatively regulates 'mitotic cell cycle'. Thus, 'cell cycle switching, mitotic to meiotic cell cycle' both positively and negatively regulates 'cell cycle'. We removed those regulation pairs since they are meaningless. (4) Compared to a feature vector for direction, which 


\section{a}

$\mathrm{p} 1 \rightarrow \mathrm{p} 2$ negative regulation pair
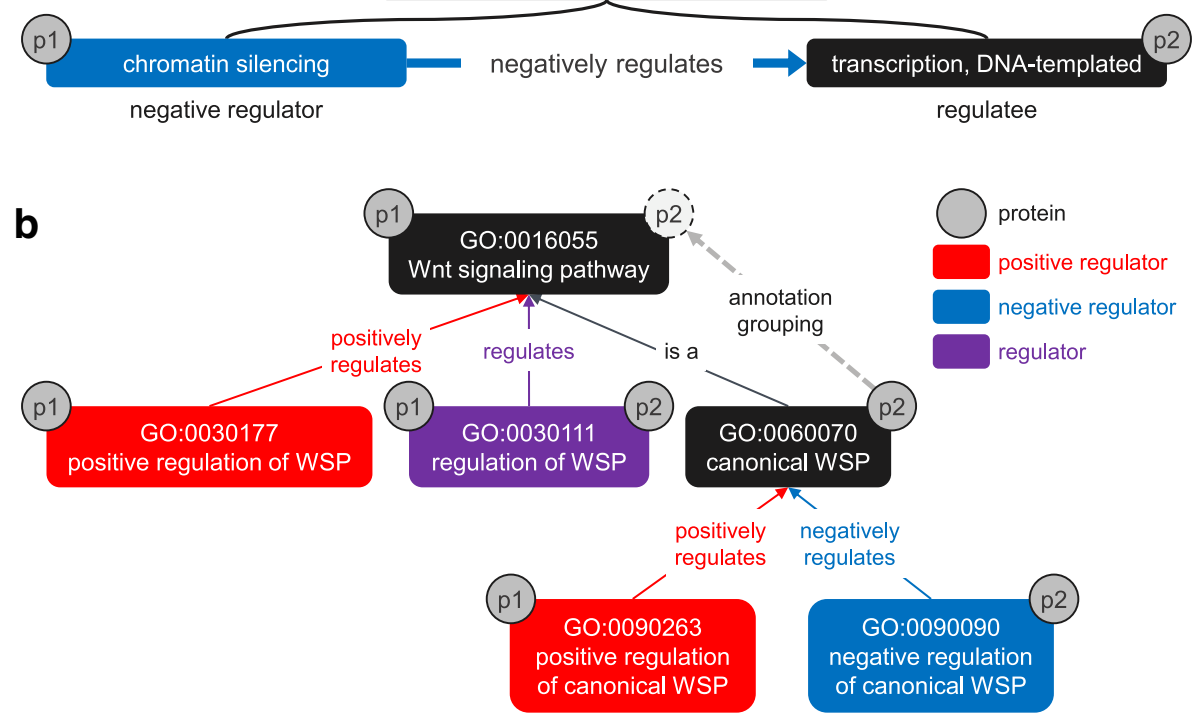
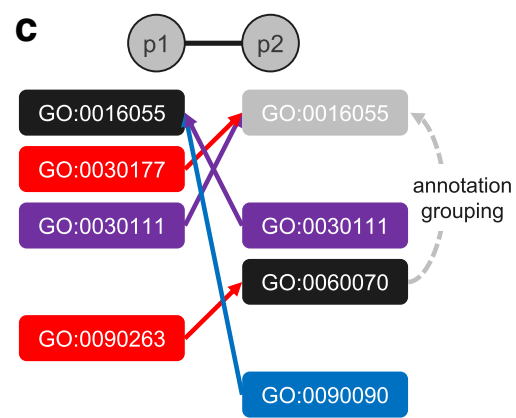

\begin{tabular}{cc}
\hline GO:0030177-GO:0016055 & 1 \\
\hline GO: 0030111-GO:0016055 & 0 \\
\hline GO:0090263 - GO:0016055 & 0 \\
\hline GO:0090263 - GO:0060070 & 1 \\
\hline GO:0090090 - GO:0016055 & -1 \\
\hline GO:0090090 - GO:0060070 & 0
\end{tabular} d

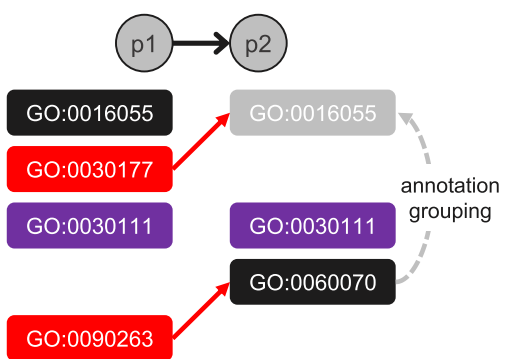

GO:0090090

\begin{tabular}{ll}
\hline GO:0030177 - GO:0016055 & 1 \\
\hline GO:0090263 - GO:0016055 & 0 \\
\hline GO:0090263 - GO:0060070 & 1 \\
\hline GO:0090090 - GO:0016055 & 0 \\
\hline GO:0090090 - GO:0060070 & 0 \\
\hline
\end{tabular}

Fig. 3 Feature generation for directions and signs. a 'chromatin silencing' negatively regulates 'transcription, DNA-templated'. Thus, 'chromatin silencing' is negative regulator whereas 'transcription, DNA-templated' is a regulatee. If protein p1 and p2 is annotated to 'chromatin silencing' and 'transcription, DNA-templated' respectively, the two GO terms compose a p1 $\rightarrow$ p2 negative regulation pair, which represents direction and sign between two GO terms. b Feature generation procedures are explained with a toy example related to Wnt signaling pathway. There are four regulators (GO:0030177, GO:0030111, GO:0090263, GO:0090090), among which two are positive regulators (GO:0030177, GO:0090263) and one is a negative regulator (GO:0090090). Protein p1 is annotated to four GO terms (GO:0016055, GO:0030177, GO:0030111, GO:0090263), whereas protein p2 is annotated to three GO terms (GO:0030111, GO:0060070, GO:0090090). Protein p2 is not directly annotated to Wnt signaling pathway', but to 'canonical Wnt signaling pathway'. Nonetheless, since 'canonical Wnt signaling pathway' is a Wnt signaling pathway', protein p2 is related to Wnt signaling pathway'. c For GO1 and GO2 to which protein p1 and p2 are annotated respectively, we determined whether GO1 (positively/ negatively) regulates $\mathrm{GO} 2$. If it did, $\mathrm{GO} 1$ and $\mathrm{GO} 2$ became a $\mathrm{p} 1 \rightarrow \mathrm{p} 2$ (positive/negative) regulation pair. If it did not, we determined whether $\mathrm{GO} 1$ (positively/negatively) regulates any ancestors of $\mathrm{GO} 2$. Then, $\mathrm{GO} 1$ and the most specific ancestor of $\mathrm{GO} 2$ became a p1 $\rightarrow \mathrm{p} 2$ (positive/negative) regulation pair. That way, we found (positive/negative) regulation pairs for $\mathrm{p} 1 \rightarrow \mathrm{p} 2$, and $\mathrm{p} 2 \rightarrow \mathrm{p} 1$ direction. To represent PPIs, we used regulation pairs as features. For directions, directions of regulation pairs were encoded as feature values. p1 $\rightarrow$ p2 (positive/negative) regulation pairs had the value of one, whereas $\mathrm{p} 2 \rightarrow \mathrm{p} 1$ (positive/negative) regulation pairs had -1 . $\mathbf{d}$ For signs, signs of regulation pairs were encoded as feature values. $p 1 \rightarrow p 2$ positive regulation pairs had the value of one, whereas $p 1 \rightarrow p 2$ negative regulation pairs had -1 . Abbreviations: GO, gene ontology; WSP: Wnt signaling pathway 
signifies the direction between GO terms, a feature vector for sign indicates signs. For predicting sign of a directed PPI $\mathrm{p} 1 \rightarrow \mathrm{p} 2$, the value of a positive/negative regulation pair $\mathrm{GO} 1-\mathrm{GO} 2$ is defined as:

$\mathrm{f}_{\text {sign }}[\mathrm{GO} 1-\mathrm{GO} 2]=\left\{\begin{array}{rr}1 & \text { if } \mathrm{GO} 1-\mathrm{GO} 2 \text { is a } \mathrm{p} 1 \rightarrow \mathrm{p} 2 \text { positive regulation pair, exclusively } \\ -1 & \text { if } \mathrm{GO} 1-\mathrm{GO} 2 \text { is a } \mathrm{p} 1 \rightarrow \mathrm{p} 2 \text { negative regulation pair, exclusively } \\ 0 & \text { otherwise }\end{array}\right.$

In our toy example, since 'positive regulation of canonical Wnt signaling pathway' and 'canonical Wnt signaling pathway' is a $\mathrm{p} 1 \rightarrow \mathrm{p} 2$ positive regulation pair, it has the value of one. On the other hand, since 'negative regulation of canonical Wnt signaling pathway' and 'Wnt signaling pathway' is a $\mathrm{p} 2 \rightarrow \mathrm{p} 1$ negative regulation pair, not $\mathrm{p} 1 \rightarrow \mathrm{p} 2$, it has the value of zero. In the toy example, since $\mathrm{p} 1 \rightarrow \mathrm{p} 2$ positive regulation pairs outnumber $\mathrm{p} 1 \rightarrow \mathrm{p} 2$ negative regulation pairs, $\mathrm{p} 1$ more likely activates $\mathrm{p} 2$ rather than inhibits $\mathrm{p} 2$. We removed regulation pairs that were not used in the training set. The number of features for sign was 20,077.

\section{Model generation for performance evaluation}

We used L2-regularized logistic regression for predicting directions and signs of PPIs. We used logistic regression because it is interpretable [20]. Moreover, L2-regularization reduces overfitting that might be caused by highdimensionality of feature vectors. As shown in Table 1, we had much more activating PPIs than inhibiting ones. To overcome this imbalance, we adopted cost-sensitive learning in which class-weight was inversely proportional to the class frequency [21].

\section{Model generation for predicting directions of PPIs}

We trained two L2-regularized logistic regression models that shared the same feature vectors for predicting directions of undirected, unsigned PPIs; they predicted whether a signal could flow in left-to-right direction and right-to-left direction, respectively. By combining outputs from the two models, we determined final directions as one of the followings: 'left-to-right', 'right-to-left', or 'bi-directional'. For example, if a signal is predicted to be able to flow in 'left-to-right' direction, but not in 'right-to-left' direction, then the final direction of PPI is 'left-to-right'. If a signal can flow in both direction, then the final direction of PPI is 'bi-directional'. Instead of training one classifier that predicts three outcomes, we trained two classifiers separately because uni-directional PPIs highly outnumbered bidirectional ones as shown in Table 1. During the training and test phase, we randomly divided unidirectional PPIs into two equal-sized sets: left-to-right PPIs, and right-to-left PPIs.

\section{Model generation for predicting signs of PPIs}

For each directed, unsigned PPI, we predicted its effect type as one of 'activation,' 'inhibition', 'activation\&inhibition', and 'affect'. Similar to directions, signs of PPIs were highly imbalanced; the number of 'activation\&inhibition' and 'affect' class were very low. Thus, we trained two classifiers, rather than a single classifier that predicts four possible outcomes. The two classifiers shared the same feature vectors and predicted whether a directed PPI can act as activation and inhibition, respectively. Then, final effect types were determined by combining outputs of two classifiers. If a directed PPI can act as 'activation', but not as 'inhibition', then its effect type were determined as 'activation', and vice versa.

\section{Results and discussions}

\section{Cross-validation performances}

We applied our method to KEGG dataset, and conducted 10 -fold cross-validation. In 10-fold cross validation, KEGG dataset is split into ten disjoint subsets. Then, we trained logistic regression models by using nine subsets, and tested the models on the remaining one subset. This procedure was repeated such that the models can be evaluated for each subset. The performance was obtained for each subset, and the mean performance is listed in Table 2. For directions, the performance of left-to-right and right-to-left classifiers were almost identical. F1-score and accuracy were as high as 0.89 for both left-to-right and right-to-left classifiers. Area under receiver operating characteristics (AUROC) and area under precision-recall curve (AUPRC) was 0.95 and 0.94 for both classifiers, respectively [see Additional file 1: Figure S1].

For signs, f1-score of activation and inhibition classifier were 0.91 and 0.80 , respectively. F1-score of activation classifier was higher because activating PPIs outnumbered inhibiting ones. Accuracy of two classifiers were identical as 0.88. AUROC were 0.94 and 0.93 for activation and inhibition classifiers, respectively. Also, AUPRC were 0.96 and 0.89 for activation and inhibition classifiers, respectively [see Additional file 1: Figure S1].

Table 2 Performance of classifiers for 10 -fold cross validation

\begin{tabular}{lllll}
\hline Classifier & Precision & Recall & F1-score & Accuracy \\
\hline Direction & & & & \\
Left2right & $0.894 \pm 0.025$ & $0.890 \pm 0.029$ & $0.892 \pm 0.027$ & $0.892 \pm 0.027$ \\
Right2left & $0.893 \pm 0.029$ & $0.892 \pm 0.023$ & $0.892 \pm 0.025$ & $0.892 \pm 0.026$ \\
Effect type & & & & \\
Activation & $0.923 \pm 0.031$ & $0.902 \pm 0.059$ & $0.912 \pm 0.040$ & $0.881 \pm 0.050$ \\
Inhibition & $0.791 \pm 0.103$ & $0.819 \pm 0.088$ & $0.800 \pm 0.077$ & $0.876 \pm 0.052$ \\
\hline
\end{tabular}

The performance is shown as mean \pm standard deviation 


\section{Independent test performances}

To see how well our model generalizes to datasets from different sources, we conducted an independent test. In an independent test, we trained logistic regression models with KEGG dataset, and tested with STRING dataset. The performance for predicting directions and signs are listed in Table 3. The performance of left-to-right classifier and right-to-left classifier were similar. Accuracy of left-to-right classifier and right-to-left classifier were around 0.6 and 0.59 , respectively. The AUROC of left-to-right classifier and right-to-left classifier were 0.64 and 0.63 , respectively [see Additional file 1: Figure S2].

The performance for predicting sign was higher than predicting directions. The accuracy of activation classifier and inhibition classifier were both 0.69. However, because the dataset was imbalanced, f1score was much higher in activation classifier than inhibition classifier. AUROC of activation classifier and inhibition classifier were 0.67 and 0.63 , respectively [see Additional file 1: Figure S2].

\section{Comparison with the previous GO-based method}

To demonstrate the effectiveness of GO relations, we compared the performance of our method to the performance of the previous GO-based method that did not use GO relations. Since the previous work did not consider directions of PPIs, we slightly modified our PPI dataset and feature values for the comparison. For PPI datasets, we originally defined effect types for directed PPIs. Therefore, bi-directional PPIs have two effect types, one effect type for each direction. To compare performance with the previous work, we mapped effect types of directed PPIs to undirected PPIs by using 'OR' operation so that bi-directional PPIs have only one effect type. For an undirected PPI p1-p2, if either directed PPIs $\mathrm{p} 1 \rightarrow \mathrm{p} 2$ or $\mathrm{p} 2 \rightarrow \mathrm{p} 1$ can act as activation, then $\mathrm{p} 1-\mathrm{p} 2$ has 'activation' as its effect type. Likewise, if either $\mathrm{p} 1 \rightarrow$ $\mathrm{p} 2$ or $\mathrm{p} 2 \rightarrow \mathrm{p} 1$ can act as 'inhibition', then p1-p2 has 'inhibition' as its effect type. The statistics of PPI dataset that were used for the performance comparison is listed in Table 4.

For feature generation, when we predict effect type of a directed PPI $\mathrm{p} 1 \rightarrow \mathrm{p} 2$, originally we considered $\mathrm{p} 1 \rightarrow \mathrm{p} 2$

Table 3 Performance of classifiers for independent test

\begin{tabular}{lllll}
\hline Classifier & Precision & Recall & F1-score & Accuracy \\
\hline Direction & & & & \\
Left2right & 0.64 & 0.60 & 0.62 & 0.60 \\
Right2left & 0.64 & 0.58 & 0.61 & 0.59 \\
Effect type & & & & \\
Activation & 0.91 & 0.71 & 0.79 & 0.69 \\
Inhibition & 0.29 & 0.49 & 0.37 & 0.69 \\
\hline
\end{tabular}

Table 4 Statistics of PPI datasets that were used for comparison with previous GO-based method

\begin{tabular}{lllllll}
\hline & & Activation & Inhibition & $\begin{array}{l}\text { Activation\& } \\
\text { inhibition }\end{array}$ & Affect & Total \\
\hline KEGG & $\begin{array}{l}\text { Previous } \\
\text { work }\end{array}$ & 14,054 & 5478 & 382 & 552 & 20,466 \\
& $\begin{array}{llllll}\text { Our work } \\
\text { STRING }\end{array}$ & 12,309 & 4787 & 369 & 517 & 17,982 \\
& $\begin{array}{l}\text { Previous } \\
\text { work }\end{array}$ & 2849 & 465 & 152 & 15 & 3481 \\
& Our work & 2729 & 455 & 152 & 15 & 3351 \\
\hline
\end{tabular}

The previous GO-based method covers all PPIs whose constituent proteins are annotated to at least one GO term. However, our method requires at least one positive/negative regulation pair to predict effect type, thereby decreasing our coverage

positive/negative regulation pairs only. $\mathrm{p} 2 \rightarrow \mathrm{p} 1$ positive/ negative regulation pairs were omitted because the direction between GO terms were inconsistent with the direction of the PPI. However, since the directions were not considered in the performance comparison, we used both $\mathrm{p} 1 \rightarrow \mathrm{p} 2$ positive/negative regulation pairs and $\mathrm{p} 2 \rightarrow \mathrm{p} 1$ positive/negative regulation pairs.

We conducted independent test for the previous work and our work. In the independent test, logistic regression models were trained with KEGG dataset, and tested with STRING dataset. The independent test results are shown in Fig. 4. For activation classifier, the performance was identical or slightly better in our method. On the other hand, in inhibition classifier, our method outperformed the previous work, especially in terms of recall. These results show that our method solved the second and third limitations of the previous work that we mentioned. The second limitation was that even if one protein inhibits another protein, the two proteins might share the same GO terms because they interact with each other. This may result in similar feature vector between activating PPIs and inhibiting PPIs in the previous work, suggesting that simply considering whether two proteins share the same GO terms are not sufficient for predicting signs of PPIs. We solved this problem by using positively regulates and negatively regulates relations in GO, not using which was the third limitation. Enhanced performance demonstrates that those relations help to predict signs of PPIs, especially for inhibiting ones.

Even though our method outperformed the previous GO-based method, our method has one drawback; slightly lower coverage. Since we need at least one positive/negative regulation pair for predicting effect types, we covered $89 \%$ of PPIs that were covered by the previous method, as shown in Table 4.

\section{Annotation of effect types to human PPIs}

We applied our method to the prediction set. We validated top five most confident predictions against 

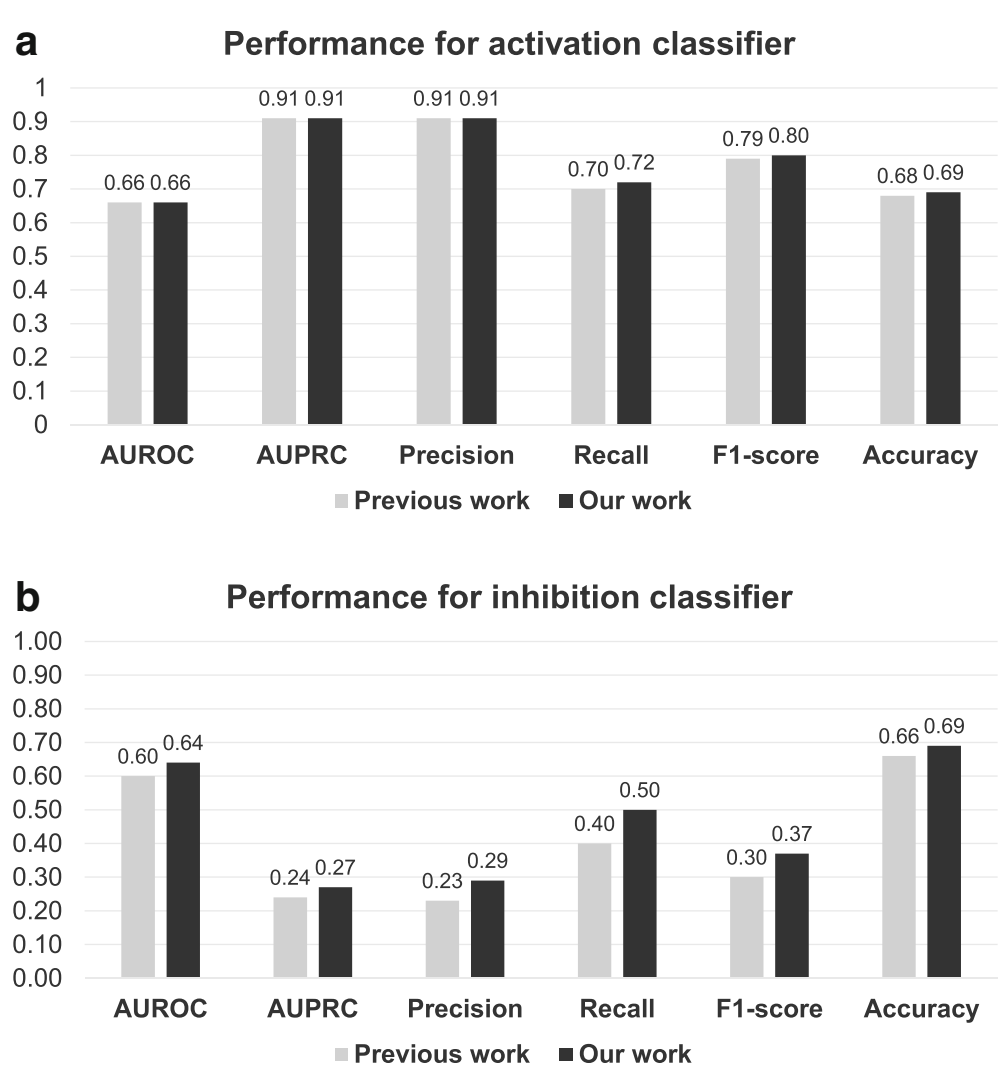

Fig. 4 Performance comparison with the previous GO-based method. We conducted independent test to compare performance with the previous GO-based method that did not consider GO relations. We compared the performance of activation and inhibition classifiers in terms of AUROC, AUPRC, precision, recall, f1-score, and accuracy. Since the previous work did not consider directions, performance for predicting signs was compared. a For predicting activation, our work performed equally, or slightly better than the previous work. $\mathbf{b}$ For predicting inhibition, our work outperformed the previous work by all metrics. These results show that overlap or difference between GO annotations of two proteins are not sufficient discriminating factor for signs of PPIs, given that inhibiting PPIs also participate in the same biological process. Moreover, positively regulates and negatively regulates relations in GO can be used for enhancing performance for predicting signs of PPIs. Abbreviations: AUROC, area under receiver operating characteristics; AUPRC, area under precision-recall curve

literature, each for activation and inhibition. We were able to find five publications supporting our prediction, as shown in Table 5.

\section{Case study}

To demonstrate that our method can predict effect types of PPIs even when one direction is activation and the other direction is inhibition, we conducted a case study. Hras proto-oncogene, GTPase (HRAS) activates mitogenactivated protein kinase 1 (MAPK1) in axon guidance, a process in which axon growth cone migrates in specific direction. On the other hand, MAPK1 inhibits HRAS in neutrophin signaling pathway. In 10-fold crossvalidation, our method correctly predicted effect types of two directions, even when one direction is activation and the other is inhibition. This means that since we predict signs for each directed PPI, and use positive/ negative regulation pairs only when their directions are consistent with the directions of PPIs, our method is able to predict effect types of PPIs independently for both directions. Regulation pairs used for the prediction is illustrated in Fig. 5. For predicting that HRAS activates MAPK1, 'positive regulation of MAPK cascade' and 'positive regulation of MAP kinase activity' were used. On the other hand, 'negative regulation of cell differentiation' was used for predicting that MAPK1 inhibits HRAS, which is related to the function of neutrophin signaling pathway. This means that some regulation pairs reflect the context in which the PPI occurs.

\section{Conclusions}

In this work, we predicted effect types of PPIs by using regulates, positively regulates, and negatively regulates relations in GO. We hypothesized that directions and signs between GO terms can be used to predict directions and signs of PPIs. For an undirected, unsigned PPI, we predicted its direction first. We trained two logistic regression models to predict whether a signal can flow in left-to-right direction and right-to-left direction, 
Table 5 Literature validation of top five activating and inhibiting PPIs from effect type-annotated human PPIs

\begin{tabular}{llll}
\hline Protein1 & Predicted effect type & Protein2 & Supporting evidence \\
\hline KIT & Activation & STAT1 & {$[24]$} \\
NRG1 & Activation & LIMK1 & {$[25]$} \\
ADAM17 & Activation & MAPK1 & \\
ABL1 & Activation & BRCA1 & {$[26]$} \\
FGF2 & Activation & RPS19 & \\
AXIN1 & Inhibition & GSK3A & \\
GRB10 & Inhibition & IRS1 & {$[27]$} \\
MAD7 & Inhibition & SMAD4 & \\
GRB10 & Inhibition & AKT1 & {$[28]$} \\
TLE1 & Inhibition & LEF1 & \\
\hline
\end{tabular}

respectively. The directions of (positively/negatively) regulates relations were encoded as features for representing direction of PPI. Then, we predicted sign for each directed PPI, thereby predicting effect type of PPI. We also trained two logistic regression models for predicting whether a directed PPI can act as activation and inhibition, respectively. We represented a directed PPI with features whose values were signs of positively/ negatively regulates relations. As a result, we annotated effect types to PPIs, thereby turning PPI network into a directed, signed graph.

Our contribution is two-fold. Firstly, we proposed a concept of $\mathrm{p} 1 \rightarrow \mathrm{p} 2$ (positive/negative) regulation pair, which is effective for predicting directions, as well as signs of PPIs. This solves the limitation of previous works that were not able to predict directions of PPIs. Secondly, we demonstrated usefulness of (positively/ negatively) regulates relations in GO. Up to date, most of GO-related works have used only is $a$ and part of relations. In this work, we showed that (positively/ negatively) regulates relations are effective for predicting directions and signs of PPIs, suggesting their extension to other types of interactions. For example, those relations might be used for predicting signs of protein-DNA interactions; whether a transcription factor activates or represses expression of a target gene.

Even though our work improved the performance for predicting signs of PPIs, we have some drawbacks. Since we need at least one positive/negative regulation pairs for predicting signs, our method has lower coverage than the previous GO-based method. We applied relation reasoning and annotation grouping to compensate low coverage of (positively/negatively) regulates relations, nevertheless, some PPIs were not covered. At this moment, our method did not consider specificity of GO terms. However, more specific GO terms have clearer meaning. This suggests that reflecting specificity of GO terms might improve our method. In addition, the performance of inhibition classifier was much lower than activation classifier since there were much more activating PPIs than inhibiting ones. This imbalance was so significant that cannot be perfectly corrected by cost-sensitive learning. We expect that accumulation of more inhibiting PPIs will enhance the performance in future.

To facilitate signaling pathway reconstruction and network biology research, we provided effect type-annotated human PPIs in Additional file 2. The annotated effect types turned PPI network into a directed signed graph, opening up opportunities for discovering new characteristics of PPI network or signaling pathways. For example, signs of PPIs can be used for measuring stability of PPI

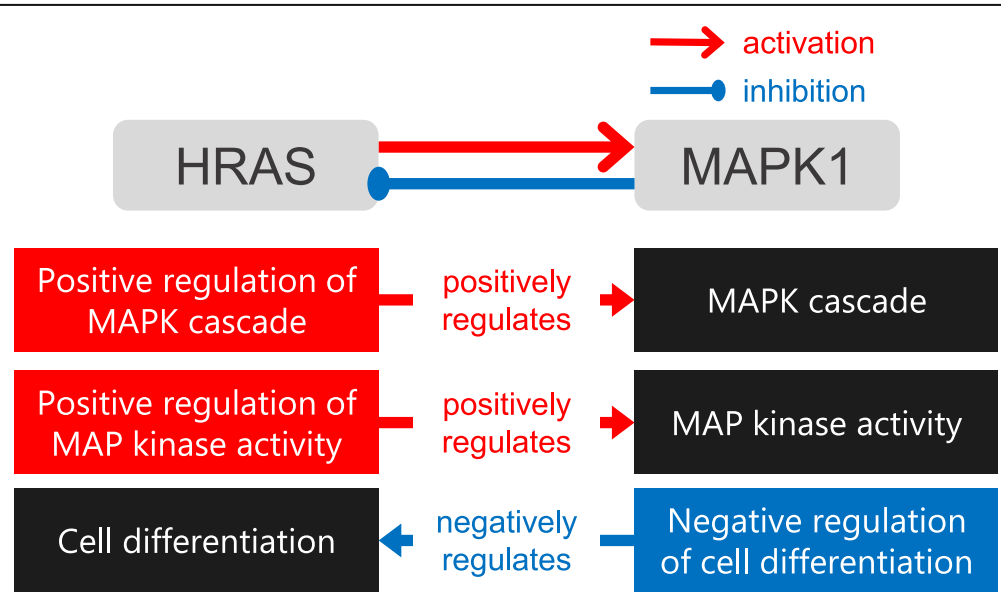

Fig. 5 A case study for PPI where one direction is activation and the other is inhibition. HRAS activates MAPK1 in axon guidance, whereas MAPK1 inhibits HRAS in neutrophin signaling pathway. Both effect types were correctly predicted in 10-fold cross validation, showing that our method can predict sign for each direction independently. Positive/negative regulation pairs that contributed to the sign prediction are shown in the figure. Interestingly, 'negative regulation of cell differentiation' and 'cell differentiation' were used to predict the inhibition, which is related to the function of neutrophin signaling pathway. Abbreviations: HRAS, hras proto-oncogene, GTPase; MAPK1, mitogen-activated protein kinase 1 
network [10, 22]. In addition, effect types of PPIs can be used for discovering novel regulators of signaling pathways [10, 23], and improving performance for predicting efficacies of drugs [8].

\section{Additional files}

Additional file 1: Table S1. Lists all possible combinations of GO relations where relation reasoning can be applied. Figure S1-S2. shows ROC and PRC along with their area under the curves obtained from cross-validation, and independent test results. (PDF $799 \mathrm{~kb}$ )

Additional file 2: Effect type-annotated human PPIs. This file contains effect type-annotated human PPIs in .xls format. Each row is a triplet of (protein 1, effect type, protein 2), in which protein 1 is a upstream protein whereas protein 2 is a downstream protein. (XLS $1547 \mathrm{~kb}$ )

\section{Abbreviations}

AUPRC: Area under precision-recall curve; AUROC: Area under receiver operating characteristics; DVL: Dishevelled segment polarity protein; GO: Gene ontology; HRAS: Hras proto-oncogene, GTPase; MAPK1: Mitogenactivated protein kinase 1; NKD: Naked cuticle; PCP: Planar cell polarity; PPI: Protein-protein interaction; RNAi: RNA interference

\section{Acknowledgements}

Not applicable

\section{Funding}

This work and publication of this article was sponsored by the Bio-Synergy Research Project (NRF-2012M3A9C4048758) of the Ministry of Science and ICT through the National Research Foundation.

\section{Availability of data and materials}

The datasets used and/or analyzed during the current study are available from the corresponding author on reasonable request.

\section{About this supplement}

This article has been published as part of BMC Systems Biology Volume 12 Supplement 1, 2018: Selected articles from the 16th Asia Pacific Bioinformatics Conference (APBC 2018): systems biology. The full contents of the supplement are available online at https://bmcsystbiol.biomedcentral .com/articles/supplements/volume-12-supplement-1

\section{Authors' contributions}

SY and DL designed this work. SY developed the proposed technique with HY and DJ under the supervision of DL. All authors read, wrote, and approved the manuscript.

\section{Ethics approval and consent to participate}

Not applicable

\section{Consent for publication}

Not applicable

\section{Competing interests}

The authors declare that they have no competing interests.

\section{Publisher's Note}

Springer Nature remains neutral with regard to jurisdictional claims in published maps and institutional affiliations.

\section{Published: 11 April 2018}

\section{References}

1. Anastas JN, Moon RT. WNT signalling pathways as therapeutic targets in cancer. Nat Rev Cancer. 2013;13(1):11-26.

2. Stewart DJ. Wnt signaling pathway in non-small cell lung cancer. Journal of the National Cancer Institute. 2014;106(1):djt356.
3. Takebe N, Miele L, Harris PJ, Jeong W, Bando H, Kahn M, et al. Targeting notch, hedgehog, and Wnt pathways in cancer stem cells: clinical update. Nat Rev Clin Oncol. 2015;12(8):445-64.

4. Yu H, Lee H, Herrmann A, Buettner R, Jove R. Revisiting STAT3 signalling in cancer: new and unexpected biological functions. Nat Rev Cancer. 2014;14(11):736.

5. Baron R, Kneissel M. WNT signaling in bone homeostasis and disease: from human mutations to treatments. Nat Med. 2013:19(2):179-92.

6. Snider J, Kotlyar M, Saraon P, Yao Z, Jurisica I, Stagljar I. Fundamentals of protein interaction network mapping. Mol Syst Biol. 2015;11(12) https://doi.org/10.15252/msb.20156351.

7. Gu H, Ma L, Ren Y, He W, Wang Y, Qiao Y. Exploration of the mechanism of pattern-specific treatments in coronary heart disease with network pharmacology approach. Comput Biol Med. 2014;51:198-204.

8. Yu H, Choo S, Park J, Jung J, Kang Y, Lee D, editors. Prediction of drugs having opposite effects on disease genes in a directed network. BMC systems biology. BioMed Central Ltd. 2016;10:17-25.

9. Yu L, Huang J, Ma Z, Zhang J, Zou Y, Gao L. Inferring drug-disease associations based on known protein complexes. BMC Med Genet. 2015;8(2):S2

10. Vinayagam A, Zirin J, Roesel C, Hu Y, Yilmazel B, Samsonova AA, et al. Integrating protein-protein interaction networks with phenotypes reveals signs of interactions. Nat Methods. 2014;11(1):94-9.

11. Suratanee A, Schaefer MH, Betts MJ, Soons Z, Mannsperger H, Harder N, et al. Characterizing protein interactions employing a genome-wide siRNA cellular phenotyping screen. PLoS Comput Biol. 2014;10(9):e1003814.

12. Mei S, Zhang K. Multi-label $\ell 2$-regularized logistic regression for predicting activation/inhibition relationships in human protein-protein interaction networks. Scientific Reports. 2016;6:36453.

13. Mei S. Probability weighted ensemble transfer learning for predicting interactions between HIV-1 and human proteins. PLoS One. 2013:8(11):e79606.

14. Yan D, Wallingford JB, Sun T-Q, Nelson AM, Sakanaka C, Reinhard C, et al. Cell autonomous regulation of multiple Dishevelled-dependent pathways by mammalian Nkd. Proc Natl Acad Sci. 2001;98(7):3802-7.

15. Kanehisa M, Furumichi M, Tanabe M, Sato Y, Morishima K. KEGG: new perspectives on genomes, pathways, diseases and drugs. Nucleic Acids Res. 2017:45(D1):D353-D61.

16. Szklarczyk D, Morris JH, Cook H, Kuhn M, Wyder S, Simonovic M, et al. The STRING database in 2017: quality-controlled protein-protein association networks, made broadly accessible. Nucleic Acids Res. 2017;45(D1):D362-D8.

17. Chatr-aryamontri A, Oughtred R, Boucher L, Rust J, Chang C, Kolas NK, et al. The BioGRID interaction database: 2017 update. Nucleic Acids Res. 2017;45(D1):D369-D79.

18. Consortium GO. Gene ontology consortium: going forward. Nucleic Acids Res. 2015;43(D1):D1049-D56.

19. Lord PW, Stevens RD, Brass A, Goble CA. Investigating semantic similarity measures across the gene ontology: the relationship between sequence and annotation. Bioinformatics. 2003;19(10):1275-83. https://doi.org/10.1093/bioinformatics/btg153.

20. Dreiseitl S, Ohno-Machado L. Logistic regression and artificial neural network classification models: a methodology review. J Biomed Inform. 2002;35(5):352-9.

21. He H, Garcia EA. Learning from imbalanced data. IEEE Trans Knowl Data Eng. 2009:21(9):1263-84

22. Anchuri $P$, Magdon-Ismail $M$, editors. Communities and balance in signed networks: A spectral approach. IEEE/ACM International Conference on Advances in Social Networks Analysis and Mining (ASONAM). ACM; 2012

23. Vinayagam A, Stelzl U, Foulle R, Plassmann S, Zenkner M, Timm J, et al. A directed protein interaction network for investigating intracellular signal transduction. Sci Signal. 2011;4(189):rs8-rs.

24. Chaix A, Lopez S, Voisset E, Gros L, Dubreuil P, De Sepulveda P. Mechanisms of STAT protein activation by oncogenic KIT mutants in neoplastic mast cells. J Biol Chem. 2011;286(8):5956-66.

25. Yin D-M, Chen Y-J, Lu Y-S, Bean JC, Sathyamurthy A, Shen C, et al. Reversal of behavioral deficits and synaptic dysfunction in mice overexpressing neuregulin 1. Neuron. 2013;78(4):644-57.

26. Maiani E, Diederich M, Gonfloni S. DNA damage response: the emerging role of $\mathrm{c}-\mathrm{Abl}$ as a regulatory switch? Biochem Pharmacol. 2011;82(10):1269-76 
27. Liu M, Bai J, He S, Villarreal R, Hu D, Zhang C, et al. Grb10 promotes lipolysis and thermogenesis by phosphorylation-dependent feedback inhibition of mTORC1. Cell Metab. 2014;19(6):967-80.

28. Hanson AJ, Wallace HA, Freeman TJ, Beauchamp RD, Lee LA, Lee E. XIAP monoubiquitylates Groucho/TLE to promote canonical Wnt signaling. Mol Cell. 2012;45(5):619-28.

Submit your next manuscript to BioMed Central and we will help you at every step:

- We accept pre-submission inquiries

- Our selector tool helps you to find the most relevant journal

- We provide round the clock customer support

- Convenient online submission

- Thorough peer review

- Inclusion in PubMed and all major indexing services

- Maximum visibility for your research

Submit your manuscript at www.biomedcentral.com/submit 